\title{
Expert based model building to quantify risk factors in a combined aquaculture- agriculture system
}

Ben Stewart-Koster ${ }^{*}$, Dieu-Anh Nguyen ${ }^{1}$, Michele Burford ${ }^{1}$, Jason Condon ${ }^{2}$, Nguyen-Van Qui $^{3}$, Le-Huu Hiep ${ }^{4}$, Doan Van Bay ${ }^{4}$, Jesmond Sammut ${ }^{5}$

${ }^{1}$ Australian Rivers Institute, Griffith University, 170 Kessels Road, Nathan, Queensland, 4111, Australia.

${ }^{2}$ Graham Centre for Agricultural Innovation, School of Agricultural \& Wine Sciences, Charles Sturt University, Wagga Wagga, NSW 2650, Australia

${ }^{3}$ Department of Soil Science, College of Agriculture and Applied Biology, Can Tho University, Campus 2, 3/2 Street, Can Tho City, Vietnam.

${ }^{4}$ Research Institute for Aquaculture No 2, 116 Nguyen Dinh Chieu, District 1, Ho Chi Minh City, Vietnam.

${ }^{5}$ Centre for Ecosystem Science, School of Biological, Earth \& Environmental Sciences, The University of New South Wales, Sydney NSW 2052 Australia

* Corresponding author: b.stewart-koster@griffith.edu.au

Keywords: Bayesian belief network, BBN, rice-shrimp farming, expert knowledge, Mekong Delta 


\section{Abstract}

In recent years, across tropical regions of the world, there has been an expansion of integrated farming systems that combine rice and shrimp production. While these systems were developed as a form of crop-rotation - growing rice in the wet season and shrimp in the dry season - some farmers grow both rice and brackish-water shrimp simultaneously during the wet season. Climatic variability has resulted in considerable crop losses in this system across many regions. Research has yet to identify the complete array of key risk factors, and their potential interactions, for integrated rice-shrimp farming. Consequently, different farming practices and environmental factors that may affect crop production need to be clarified to guide research efforts. We applied a staged, iterative process to develop a probabilistic Bayesian belief network based on expert knowledge that describes the relationships that contribute to the risk of failure of both crops in integrated rice-shrimp farming systems during the wet season. We applied the approach in the Southern Mekong Delta, Vietnam, in the context of a broader research program into the sustainability of the rice-shrimp farming system. The resulting network represents the experts' perceptions of the key risk factors to production and the interactions among them. While both farmers and extension officers contributed to the identification of the processes included in the network, the farmers alone provided estimates of the probability of the relationships among them. The network identified the challenges to minimise the risk of failure for both crops, and the steps farmers can take to mitigate some of them. Overall, farmers perceived they have a better chance to minimise risk of failure for shrimp rather than rice crops, and limited opportunities appear to exist for successful production of both. By engaging the farmers in this process of model development, we were able to identify additional research questions for the broader research team and to identify simple steps the farmers could take to reduce the risk of crop failure. Integrating additional empirical data into this network, as it becomes available, will help 
identify clear opportunities for improvements in farming practices which should reduce the risk of crop failure into the future.

\section{Highlights}

- We converted knowledge from rice-shrimp farmers in the Mekong Delta into a probabilistic model

- Key risks to production for both crops revolved around soil and water quality

- On average, rice crop failure was perceived to be more likely than for shrimp

- Capturing extant agricultural knowledge improves outcomes for research and practice 


\section{Introduction}

Agricultural production is affected by numerous environmental factors, some of which are within the control of producers and others that are beyond their control. Human alteration of the environment and the increasing impact of climate change combine to increase risks of crop failure in many regions across the world (Morton 2007). The risk of recurrent crop failures is higher for integrated aquaculture-agriculture systems in landscapes influenced by brackish waters where rising sea levels and altered freshwater inflows combine to modify hydrological conditions throughout the growing cycle (Huq et al. 2015). These combined altered processes affect the hydrological and thermal regimes, ultimately influencing salinity and nutrient concentrations in pond and canal waters (Thanh et al. 2004). Subsequently, individual farmers make decisions on crop management in an attempt to reduce risks of crop failure and financial losses (e.g. Everingham et al. 2002).

Understanding and quantifying the multiple stressors affecting production, including impacts of decisions by individual farmers, is an important step to minimise risks of crop failure (Reid and Vogel 2006). In relatively well-studied systems this may already be well formalised following years of research and production. However, in emerging agricultural regions, such as those in the developing world, this understanding may not yet be widely established (Godfray et al. 2010). In developing regions, documenting existing knowledge held by farmers in a systematic manner provides an avenue to incorporate such expertise into userfriendly decision support tools (Walker et al. 1995). This is particularly important given decision making by farmers is grounded in a broader socio-economic system that can be difficult to integrate in agricultural research (Joffre et al. 2015). With the development of such tools, findings from complementary empirical research are more likely to be of use to 
participating farmers and can complement their existing knowledge and understanding (Kristjanson et al. 2009).

The field of participatory modelling has developed to integrate the existing knowledge and expertise of practitioners into empirical research (Voinov and Bousquet 2010). Such expertise may be in the form of traditional ecological knowledge of local and indigenous communities, lifelong experience of farmers or other knowledge developed through experience of living and working in a given system. Depending on the context, experts may be farmers, local indigenous people, scientists or other experienced practitioners. Integrating agricultural expertise allows the development of a link between science and farmers, enabling them to contribute actively to relevant research. This has several advantages including the identification of issues of research interest that are most crucial to farmers which ensures any empirical research is specifically relevant to farmers and farm management. When participatory modelling engages farmers and policy makers, it helps establish and foster a link between policy and on-farm decision making (Joffre et al. 2015), the lack of which can lead to negative outcomes for regional farmers. One avenue of such modelling is the development of probabilistic models that can integrate expert knowledge with existing data and knowledge via Bayesian belief networks (BBNs), a probabilistic graphical modelling tool.

Bayesian belief networks provide a very powerful tool for management and decision-making in agricultural settings (Cain et al. 2003; McCann et al. 2006). They have been used widely in many different natural resource management contexts because of their inherent flexibility and capacity to quantify complex social, economic and ecological relationships (McCann et al. 2006; Stewart-Koster et al. 2010; Phan et al. 2016). By using BBNs, it is possible to develop a probabilistic model that reflects the conceptual understanding of the system under 
study in the form of an influence diagram; this can then be populated with the available data from multiple sources, including empirical data and expert knowledge (Korb and Nicholson 2004). This approach can be applied where empirical data may be lacking but where considerable expert knowledge exists in the form of traditional ecological knowledge or accumulated experience of farmers and scientists (Liedloff et al. 2013). Such knowledge can be incorporated into the model to form the network structure and the relationships it quantifies.

Developing BBNs from expert knowledge requires a systematic expert elicitation process, usually involving a series of workshops, to formalise the knowledge and experience into a suitable model (e.g. Johnson et al. 2010; Bashari et al. 2009). The goal of such a process is to extract information from experts while overcoming potential pitfalls associated with bias and subjectivity (Kuhnert et al. 2010). Such pitfalls include strong personalities dominating the process and subsequent outputs, unclear statements of the causal relationships in the system, and language barriers leading to misunderstandings between BBN developers and experts (Renooij 2001). Failing to account for these issues can lead to the development of a BBN that does not accurately reflect the system and the experts' understanding of it, potentially leading to poorly informed decision making (Kuhnert et al. 2010).

There are many different workshop approaches to develop BBNs based on expert knowledge or opinion (e.g. Smith et al. 2005; Ticehurst et al. 2007; Johnson et al. 2010; Schmitt and Brugere 2013). These workshops may involve various numbers of experts, though typically a small group is engaged, for example, Schmitt and Brugere (2013) relied on twelve experts to fine tune a BBN for shrimp farming in Thailand. In some cases experts may spend several days with workshop facilitators and BBN developers (e.g. Smith et al 2005; Johnson et al. 2010), while in others such elicitation may be done over email (Kuhnert et al. 2010). The 
process may focus solely on developing the network structure or include the additional step of deriving the strength and nature of the relationships within the network. Importantly, several stages of validation are included to identify any miscommunications and ensure the resulting network accurately reflects the knowledge and understanding of the participating experts, which may be particularly important when language barriers are present during the process (Johnson et al. 2010; Kuhnert et al. 2010). These approaches have been very successful in developing BBNs across a range of systems, however, they require a substantial time commitment by the experts, which is something that may not always be available. These kinds of demands on experts can create a challenge for the development, implementation and uptake of the BBNs by potential end users. Additionally, making use of only a small number of experts may lead to a narrow view of a system that does not reflect the broader understanding that may exist within the given community (Kuhnert et al. 2010).

Given the potentially prohibitive time commitments required of experts for BBN development, it is clear that an expert elicitation that requires less time in any one sitting would be advantageous. We developed a staged process of BBN development to incorporate the expertise of multiple groups of experts in relatively short workshops. We applied this process to quantify key risk factors to production faced by local farmers in the Mekong Delta, Vietnam, who practice integrated rice-shrimp farming during the wet season when conditions may not be optimal for both species simultaneously. The experts consisted of local farmers and government extension officers from three neighbouring provinces, who were participating in a broader research program into the sustainability of rice-shrimp farming systems. Much of the region has been converted to rice-shrimp farming during the previous 10 years and already is facing severe risks to production, leading in some cases to crop losses, from anthropogenic and climatic factors. However, research has yet to identify key risk factors, and their potential interaction, for both rice and shrimp farming. Consequently, the 
process and effects of different farming practices and environmental factors need to be elucidated to guide the ongoing research.

Our process was based on the Iterative Bayesian Network Development Cycle, developed by Johnson et al. (2010), to ensure the input of each group of stakeholders was cross-validated by all others. Consequently we derived a regionally applicable and internally-validated BBN based on the expert knowledge of farmers across several provinces. The final network model provides an avenue to incorporate empirical data on processes affecting farm production that are identified as relevant to the farmers themselves. This also provides an avenue to engage farmers in ongoing research and identify key knowledge gaps among farmers that will provide the greatest opportunity for improvements to production.

\section{Materials and Methods}

\subsection{Study setting: Rice-shrimp farming}

Integrated rice-shrimp farming in coastal regions is an integrated agricultural system that emerged as a mechanism to derive some production from fallow rice fields during the dry season when water salinity rises with decreased freshwater flows and rainfall. Salinity also increases due to higher evaporation during the dry season. Typically, rice is sown on a platform for wet season production with a deeper ditch surrounding the platform in which shrimp may be raised simultaneously if conditions allow. During the dry season, when water salinity rises, production moves to shrimp that inhabit both the ditch and rice platform. We developed the BBN for rice-shrimp systems with farmers and extension officers from three

provinces of the Vietnamese Mekong Delta; Ca Mau, Bac Lieu and Kien Giang (Figure 1a). While relatively close in proximity, farms in each of these regions experience slightly different hydrologic regimes due to variation in freshwater inflows from upstream and 
regional infrastructure to control such flows. We focused on the wet season because of the evident challenges faced by farmers of growing a freshwater crop, such as rice, in tandem with a brackish shrimp species.

\subsection{Bayesian belief networks}

A BBN is a graphical model consisting of nodes and links where nodes represent the key factors or processes of a given system and links represent the causal relationships among them (Korb and Nicholson, 2004; Jensen and Nielsen, 2007). Links in a BBN are unidirectional and connect 'parent nodes' to 'child nodes' which results in no feedback loops that would make child nodes become parent nodes of their ancestral nodes (Jensen \& Nielsen, 2007). Each node is defined categorically having multiple possible states (e.g. high, medium, low), with the probability of each state of a node dependent on the states of parent node(s). These probabilities are defined by the conditional probability table (CPT) of each node. The CPTs may be derived from a range of different sources including empirical data, expert opinion or results from previous research (Korb and Nicholson, 2004). A key assumption of BBNs is known as the Markov property, which means that the CPT of a node is dependent on the immediate parent nodes only. When using expert opinion to populate the CPTs of a BBN, this can help simplify the elicitation process, because experts only need to consider the states of the immediate parent nodes and not the full complexity of the network.

\subsection{BBN development}

The process to develop the BBN involved several stages of targeted expert elicitation workshops with extension officers and farmers in each region forming the groups of experts whose knowledge and experience populated the network. We conducted three research trips in October 2013, July 2014 and November 2015 during each of which, somewhat atypically 
for a BBN development process, we could only spend a matter of hours with each group of experts. As such, we included an iterative process of validation at each stage of the elicitation to ensure specific regional biases did not affect the outcome of the BBN (Figure 1b).

The entire process began with a one-day introductory training workshop for facilitators (Vietnamese researchers from partner organisations, including the Research Institute for Aquaculture 2 (RIA2), Can Tho University (CTU) and Cuu Long Rice Research Institute Vietnam (CLLRI)), who would work with the BBN modellers to interact with the farmers and policy officers as facilitators and translators. The preliminary training workshop focused on the theory and application of BBNs and culminated in groups of researchers developing and presenting their own BBN for scrutiny by other workshop participants. This process ensured the training worked in two directions, the newly trained facilitators had a clear understanding of the BBN development process from beginning to end, and the BBN trainers and modellers were introduced to likely important environmental and agricultural processes, as perceived by the facilitators. Understanding the processes that were viewed as important by the facilitators also helped identify possible biases they may have unconsciously focused on in the subsequent expert elicitation workshops. Given the language barrier between the modellers and the experts, these biases may have otherwise been missed.

\subsection{Defining nodes and network structure}

The first two stages of the BBN development process began with eliciting risk factors for the individual rice and shrimp production processes from the first groups of experts in Ca Mau province. With the second two groups of experts, from Bac Lieu and Kien Giang provinces, we sought to validate the risk factors and rapidly identify others that may be relevant before prioritising key factors to determine nodes for the network and the relationships therein. 
Rather than asking experts directly to arrange the nodes and define child and parent nodes, facilitators framed questions about how farmers expect certain nodes to change should a key process change (for example, under what conditions does soil salinity change?), or how the farmers react under certain conditions (for example, what do you do if you perceive the soil quality to be poor at the start of the season?). The answers to the full range of these questions identified the connections between child and parent nodes.

Selecting thresholds for the different states in a node is an important process in BBN development. In many contexts these can be defined by scientifically defined boundaries, for instance established lethal thresholds can be chosen for physical parameters such as salinity and dissolved oxygen concentration (Marcot et al. 2006). In this context it was important to consider the capacity for objective measurement and monitoring on the part of the farmers. For example, water salinity is monitored by taste rather than any kind of salinity probe. As such, thresholds for physical parameters such as salinity were defined against the famers' expectations of rice or shrimp health. Moderate dissolved oxygen concentrations, for example, being sub-lethal concentrations that would create stress on shrimp and low dissolved oxygen concentrations that which would lead to mortality. For composite nodes, such as rice platform preparation, farmers were asked simply subjectively about what they considered Good, Average or Poor, and in this way the probability distributions represent a range of experience with associated uncertainty.

\subsection{Estimating conditional probability tables}

Having identified key processes (nodes) and relationships among them (links) on the first research trip, we began the process of populating the CPTs based on the expert opinion of only the farmers, in all three regions during the second research trip (Stage 3 in Figure 1). This stage began with a second training workshop to ensure workshop facilitators were 
familiar with different techniques of probability elicitation. This included a series of approaches including; a survey method, direct probability elicitation and indirect approaches such gamble-like methods (Table 1). Each of these approaches has its own strengths and weaknesses, and presents unique challenges (Renooij 2001). Generally different techniques are used for different circumstances. In our workshop process, we applied different approaches depending on the nature of the child node of interest.

The survey method we developed involved separately asking ten or more experts to state the most probable state of a child node under a given set of parent node states. The frequency that each state in the child node was chosen as the most probable outcome under each set of conditions provided the probability of that outcome in the CPT. This approach was used for child nodes with large CPTs (i.e. nodes with many parent nodes or parent nodes with many states). For such CPTs, experts found it easier to state a single most probable state, rather than a probability estimate for all states, under given values of the parent nodes.

Direct methods of probability elicitation focused on asking experts to estimate the probability of each state of the child node, given specific states of relevant parent nodes. This approach tended to be most useful where there was an easily visualised state in the child node, such as proportions of a crop or farm area, that the farmers expected would have a certain state given parent node conditions. For example, under given water quality conditions, what proportion of a shrimp crop would show high, moderate and low levels of stress? This approach was also useful for relatively small CPTs, being nodes with few parent nodes.

Finally, we trialled a gamble-like method for the terminal nodes where experts were asked to choose to bet on a hypothetical lottery with known odds or bet on the risk of crop failure under certain parent node conditions. Varying the known odds of the hypothetical lottery until the experts change their choice of bet identifies the expected probability of the outcome 
under consideration (Renooij 2001). Unfortunately we had to abandon this approach because several of the participating farmers refused to ever bet against their own rice crops, no matter the odds of winning a lottery!

\subsection{Validation and verification}

The final step involved a staged process of verification of the entire network and the CPTs governing the relationships. This began with a presentation of the structure of the entire BBN to the farmers for validation, and subsequently working through the network to present the probability of child node states under different parent node conditions. To facilitate the verification component of this process, we worked with each group of farmers on the nodes and CPTs they themselves had defined. To facilitate the validation component, we also presented key nodes and CPTs that were developed by farmers from different regions. This ensured that there was validation from farmers both within and across regions within the study area.

The approach to this stage of the BBN development process was to show the farmers graphically, how the probability of each state of a given child node changed under different states of the parent nodes (Figure 2). Conditions were set for specific parent nodes within the BBN which illustrated the expected probable outcomes of the child node under discussion. For example, when platform preparation is of good quality and rainfall volume is high, the CPT suggests there is a $100 \%$ probability of low soil salinity (Figure 2a). When rainfall volume is set to low, there is a $75 \%$ probability of high soil salinity and $25 \%$ probability of moderate soil salinity (Figure 2b). The farmers were invited to alter outcomes using the graphical illustration of the CPTs, where they disagreed with them. All CPTs that were changed in the verification stage were presented to additional experts to ensure a single farmer, or group of farmers, did not have undue influence on certain components of the 
network. As a result of this step, the final workshop involved verification of previously altered CPTs only.

Before the validation phase began, the BBN modellers explored probable outcomes under the scenarios of entire model to identify any unexpected or unlikely outcomes. These may have come from misunderstandings in complex CPTs or even mistranslations between the experts, workshop facilitators and BBN modellers. Where these outcomes were found (e.g. highly saline soil leading to low risk of rice failure), they were replaced once clarified in the validation phase. Where there were more subtle differences among experts in the probabilities obtained, an average was taken to complete the CPT.

This staged approach to elicitation ensured the final BBN represented all regions in the study area while accommodating locally-specific relationships where necessary. In total 32 farmers and 26 extension officers across all three provinces participated in the expert elicitation process for the BBN.

\subsection{Additional data used in the model}

In addition to the expert knowledge of the participating farmers, we also used available rainfall data to define the CPTs for nodes relating to the onset of the wet season and the volume of early wet season rainfall, as these were parent nodes only. These data consisted of daily rainfall records for 21 years (1976-2006), obtained from the Ca Mau monitoring station (www.gso.gov.vn).

Discussions during the workshops highlighted that the wet season was generally considered to begin in May each year. As such, an early onset was defined as a year when the total April rainfall was more than one standard deviation greater than the long term mean total April 
rainfall. A late onset was defined as a year where April rains were not above normal and May rainfall was more than one standard deviation less than the long term mean total May

rainfall. An average onset was defined as a year when April rains were not above normal and May rains were within one standard deviation of the long-term mean total May rainfall. The volume of early wet season rainfall was defined similarly, using the total rainfall for May and June to define early season rains as High, Low or Average volume. Average volume was defined as the years in which total rainfall for May and June was within one standard deviation of the long-term average, while High or Low years were those one standard deviation above or below the mean. We calculated the frequency of each of these outcomes based on the 21 years of data to populate the CPTs for the two rainfall nodes.

The network was constructed using the BBN software, Netica (V4.16, Norsys, 2010).

\section{Results}

The final BBN comprised a collection of nodes representing processes of climate/weather, soil and water, rice and shrimp as well as management/decision options available to the farmers, all contributing to determine the risks of rice and shrimp crop failure in the wet season (Figure 3). A top-down perspective of the BBN reveals that the entire system is perceived to be governed by the timing and duration of wet season rainfall, which determine the "wet season type". This outcome contributes to the states of multiple water quality nodes, as well as influencing soil quality and the quality of platform preparation for rice-sowing. Management options available to the farmers were perceived to occur throughout the BBN, and included processes such as preparation of the rice platform to influence soil and water quality, or adjusting fertiliser application in response to rice crop growth. Several composite nodes, such as soil quality and water quality, were created to summarise complex processes into a single outcome. The terminal nodes, risk of failure of each of rice and shrimp crops, 
had states referring to levels of risk of failure (high, medium and low) as well as a state for complete crop failure.

The structure of the BBN identified several interactive connections between the conditions that influenced each crop (Figure 3). The soil quality of the rice platform, which was directly connected to risk of rice failure, was perceived to be a function of platform preparation and wet season rainfall conditions. Not surprisingly, wet season rainfall had a direct bearing on water quality; however, the farmers and extension officers also identified a clear relationship between water and soil quality. Water quality was defined as being either good for rice, shrimp, both or neither, dependent on soil quality, dissolved oxygen and the salinity and nutrient content of the water. In addition, perceptions of the soil quality had a direct bearing on the stocking rate of the shrimp in the ditch around the platform. With all these interactive relationships established, the farmers showed contrasting expectations of risks to rice and shrimp crop failure.

With no conditions set in the BBN, the probabilities for each state in each node represent the averages (as in Figure 3). Overall, the farmers had a generally pessimistic outlook on their rice crops, with an average of $61 \%$ probability of crop failure and $30 \%$ probability of being in conditions of high risk of crop failure (see terminal node for 'Risk of rice failure' in Figure 3). In contrast, they were more optimistic about the risks of the failure of a shrimp crop with a split of $28 \%$ probability of crop failure and approximately $48 \%$ probability of low risk of crop failure (see terminal node for 'Risk of shrimp failure' in Figure 3). Interestingly, the farmers were also pessimistic about the growing conditions with an expectation of a 57\% probability of water quality being neither good for rice nor shrimp (such conditions would be highly saline, potentially very warm with very low dissolved oxygen). In conjunction with this was a 34\% probability of water quality being good for shrimp. 
Setting the conditions of each of the child nodes to low risk of crop failure reveals the conditions under which the farmers perceive those outcomes to be possible (Figure 4). The most striking aspect of this is that these joint outcomes are only possible when water quality is good for rice growing (see Wet Season WQ node in Figure 4a). These conditions are expected to occur when the wet season has either average or high rainfall (see nodes for Rainfall Volume and Wet season type in Figure 4a). In contrast, setting both child nodes to crop failure, shows that these outcomes may occur under any water quality conditions, but are most likely when it is good for shrimp (Figure 4b). Compared with the low risk settings for the child nodes in Figure 4a, the relatively even probability distributions on the ancestral nodes of Figure 4b suggest that there are more scenarios that will lead to crop failure.

Sensitivity analyses of the two child nodes in the BBN identified the ancestral nodes that have the greatest influence on their states (Table 2). Parent nodes that were directly connected to the child nodes of interest, such as the parent node water quality and for the child node risk of rice failure, were found to be the most influential. Nonetheless, several ancestral nodes were also influential on the child nodes, for example soil and water salinity were the third and fourth most influential nodes for rice failure. Interestingly, several nodes over which the farmers have some direct control via management options were highly influential nodes such as fertiliser regime, platform preparation and soil quality as well as shrimp stocking density and shrimp stress detection (Table 2). This information identifies processes over which they have control that the experts collectively perceive as important, as opposed to important processes over which they have no control such as the nature of the wet season.

The impact of influential nodes can be visualised by setting conditions for them and inspecting the outcome on the child nodes (Figures 5 and 6; Appendix A). The importance of 
water quality is evident with a shift from generally low risk of rice crop failure when conditions are set to being good for rice, to an expectation of complete crop failure when water quality is set to be good for shrimp (Figures 5a and 5b). Regardless of whether platform soil quality is either good or poor, farmers had some expectation of possible crop failure (Figures $5 \mathrm{c}$ and $5 \mathrm{~d}$ ). The most evident change was that good soil quality led to a greater expectation of low risk of crop failure. These patterns held in the shrimp component of the network with conditions of high or low shrimp survival clearly affecting the probability of different levels of risks to crop failure (Figures 6a and 6b). Even when survival is high the farmers perceived there to be a 7\% probability of crop failure (Figure 6a). This pattern is evident with the presence or absence of disease. When disease is present, the farmers have a generally high expectation of crop failure (Figure 6c). When it is absent, risks to crop failure decline, however, there is still almost $20 \%$ probability of crop failure due to other factors in the BBN (Figure 6d), which would include outbreaks of benthic algal mats (lab lab) or general stress due to poor water quality (Figure 3).

\section{Discussion}

We successfully applied a process to integrate the knowledge and experience of multiple experts, including farmers and extension officers, into a single probabilistic model that describes the risk factors to a common tropical agricultural system. Our staged approach of network development ensured we applied what we learned from each group to subsequent groups in the study, in an iterative model building process. The contributions of the experts identified key factors that affect risks to crop production, both environmental (i.e. things that can't be controlled) and agricultural (things that can be controlled). By introducing conditional questions grounded in the already forming network we successfully captured the structural connections in the BBN, which led us to potential decisions made by the famers in 
response to the changing environment in which they work. Subsequently, the final BBN looks different from our initial expectations; importantly the farmers were all in general agreement about its accuracy in reflecting their understanding of the system.

The final BBN developed here identified a complex series of interactions among environmental and agricultural factors. The network indicates that farmers generally perceive it to be very difficult to balance the needs of both crops and reduce the risk of crop failure for both simultaneously. Nonetheless, conditions can occur where this is possible even when the preparation for sowing of rice and the quality of shrimp seed stock is not optimal. As such, the BBN provides an avenue to guide decision making at the individual farm level, given a range of environmental conditions.

The BBN captures the many complex interactions that influence the probability of crop loss in a given season. Many of the variables (nodes) in these interactions can be measured directly by the farmers, albeit imperfectly in the case of tasting the water to estimate salinity, however, several cannot be measured, such as dissolved oxygen. Nonetheless, the network can be used to assist farmers in their decision making both before and after planting and stocking as there are multiple management decision nodes in the BBN (liming of soil, tilling of soil, fertiliser application, shrimp stocking density and management of water colour and water quality for shrimp stress). For example, an assessment of the nature of the early stage of the wet season will give a farmer an indication of the quality of platform preparation they will likely be able to complete along with the probability of different water quality conditions. This can help guide the decision about if and when to sow a rice crop. Guidance on within season decisions such as how much fertiliser to apply given the appearance of the rice crop and an assessment of the soil quality is also available with an estimated probability associated with each outcome of the child node. 


\subsection{Integrating expert knowledge and data in BBN development}

The processes that emerged from the workshops were generally consistent with available scientific evidence of processes that affect rice-shrimp farming in the region. The farmers had a clear understanding that soil quality, and in particular soil salinity, played a crucial role in the risk of crop failures. As well as being a known risk factor for rice production, high levels of soil salinity may lead to considerable land degradation in this region (Tho et al. 2008; Hens et al 2009). Equally, the farmers understood that water quality, including parameters they could not directly measure, such as dissolved oxygen, are important factors that contribute to the risk of failure of both crops. These have been found to be at critical levels for shrimp in the integrated rice-shrimp farming systems of this region (Tho et al. 2011; Leigh et al. 2017). Nonetheless, there are additional factors that were not identified through the BBN workshops that may be risk factors for the crops, including the composition of the biological community of the rice-shrimp ponds. These have been found to include toxic forms of cyanobacteria and relatively low levels of zooplankton and algae upon which growing shrimp can feed (Tho et al. 2012). The next phase of model development will include the use of available data on such additional factors. Additionally, experimental and observational data collected in the research accompanying this BBN will be integrated into this BBN to identify important areas of learning (Ticehurst et al. 2007).

An important feature of BBNs is that information from multiple sources can be used to define the nature of the relationships in the nodes (Korb and Nicholson 2004). In the present context, that could involve incorporating data from ongoing experimental research into the existing BBN to complement the farmers' knowledge. In such a case the existing CPTs collected from the farmers’ knowledge could form Bayesian prior distributions for use with new data (Pollino et al. 2007). Additionally, this would provide a basis to "validate" the 
understanding of farmers against experimental data (Ticehurst et al. 2007). Alternatively, a revised hybrid BBN could be developed, beginning with the farmers' expert knowledge and integrating additional nodes and links defined according to experimental research or information from the literature (e.g. Landuyt et al. 2014). In addition to making use of all available information in model development, such an approach would assist in highlighting effective opportunities for research extension. The CPTs that change dramatically with the addition of experimental data to the prior information from the farmers would be those where the farmers' knowledge differs most substantially from research findings. These are the areas where considerable improvements may be made in farming practice given the research findings.

\subsection{Reaching a collaborative research agenda}

Bridging the gap between researchers and agricultural decision makers and farmers is a major step toward sustainable development (Kristjanson et al. 2009). A major advantage of this approach for research in agricultural systems is that it engaged the participating farmers in the research process (Cain et al. 2003). This provided an opportunity for the farmers to contribute to the agenda of the broader research effort by identifying processes that were not previously considered by the research team. Additionally, the BBN workshops highlighted potential decisions farmers may make to improve conditions in the rice-shrimp system in an attempt to reduce the risk of crop failure. Subsequently, the broader research team were able to tailor experiments that were specifically relevant to the management decisions available to the farmers in the system. For example, it emerged during the workshops that farmers in one region had recently begun tilling the soil in advance of rice sowing. However, with limited experience in this practice the farmers were unsure of its potential to reduce risks of crop failure. Consequently, the practice of soil tilling has been incorporated into the experimental 
work of the broader research program, the results of which will feed directly into the BBN and contribute to improving the farmers’ practice in this system.

In addition to engaging farmers in the research process, developing a BBN with practitioners can provide immediate gains for the participants. During informal follow up interviews about their understanding of the network, a subset of farmers described how they had learned different approaches and ideas from the process, based on the discussions with others in the workshops. These farmers had already slightly changed their practice, in particular regarding soil preparation and water quality monitoring, and were looking forward to findings from ongoing experiments.

\subsection{Bayesian belief networks in agricultural research}

There are clearly several advantages to the use of BBNs in agricultural research, particularly in relatively under studied systems, such as the rice-shrimp production system in the Mekong Delta. However, a caveat of this approach is that there is a great deal of opportunity for misunderstanding during model development (Renooij 2001; Chen and Pollino 2012). The language barrier is an ever present challenge in contexts like ours (Kuhnert et al. 2010), as is the lack of familiarity with a modelling approach, such as BBNs, on the part of the experts. We attempted to circumvent these issues with three strategies. The first was to ask indirect questions about causal relationships and decisions farmers were likely to make over the course of the growing season, so as to define the network structure indirectly (Kuhnert et al. 2010). This helped to ensure workshop facilitators did not ask leading questions and the answers were those of the farmers themselves. The second strategy was to use different probability elicitation techniques for different types of nodes (Renooij 2001). Where it was feasible to think of probability in terms of proportions, such as shrimp survival, we asked the farmers to think in those terms (for example, what proportion of the crop would survive under 
given conditions). In contrast, for nodes where calibration by the farmers was difficult due to a lack of scientific instruments, such as water salinity, we encouraged experts to think in terms of impact on a crop (e.g. the state of high salinity, identified by tasting pond water, would be that which damages rice and shrimp). The third strategy was to incorporate multiple stages of verification where different facilitators verified network structure and the CPTs with different farmers from those who defined them. These steps ensured the final network was a broad consensus model that represents our best interpretation of the experts' understanding of the system.

A feature of many applications of BBNs is that they may be context specific, with network models often being applicable for the setting in which they were developed for local use only (e.g. Ticehurst et al. 2007; Rahman et al. 2015). This is a clear advantage because the network can represent very specific factors and quantify probabilistic responses to locally relevant causal processes (McDowell et al. 2009). However, the lack of generality can limit the transferability to related systems with slightly different contexts. Our approach of drawing on experts from multiple regions, including for model validation, helped to ensure the potential for regional application of the network. The factors that emerged as important tended to be physical processes, which may be more general and therefore apply in other locations. Developing BBNs that are more broadly relevant can provide farmers and policy makers with a decision support tool that applies beyond a small geographic region. This is particularly plausible where physical processes that are not context dependent are modelled. Multiple sources of data, including experimental research data from related systems, can subsequently be incorporated with the expert opinion.

\subsection{Conclusion}


With farmers in marginal agricultural lands facing an ever-growing list of factors that increase the risk of crop failure, innovative modelling techniques are increasingly relevant to ensure the research process leads to tangible benefits for crop production and land management. A process such as that presented here provides numerous benefits to engage farmers in the research process and develop a netwok model that is specifically relevant to them. The network itself includes farm-level factors, such as soil and water salinity, as well as larger scale factors that are outside the control of the individual producer but are vital constraints on production, such as rainfall conditions. Given the expected changes to these factors due to climate change and increasing flow diversion of the worlds' rivers, quantifying their impact on risks to production and identifying decisions available to local farmers to mitigate them, becomes increasingly important. While not a panacea, research and modelling that engages farmers in the construction of probabilistic models of their agricultural systems offers a great step forward.

\section{Acknowledgements}

The authors would like to thank all of the farmers and extension officers who gave their time and energy in contributing their knowledge and experience for this research. We thank Dr Nguyen Van Hao, Mr Nguyen Cong Thanh and Ms Hoang Thi Thuy Tien for help coordinating all of the workshops. We also thank Mr Luu Duc Dien and Dr Pham Cu Thien for help and facilitation during the workshops. We thank Graeme Curwen for producing the map in Fig. 1. This research was conducted under a Griffith University Human Ethics permit (ENV/28/14/HREC). Funding for this work was provided through an Australian Centre for International Agricultural Research (ACIAR) project grant (SMCN/2010/083). The second training workshop was funded by an individual training award from the Crawford Fund (QLD-622-2013). 


\section{References}

Bashari, H., Smith, C., Bosch, O.J.H., 2009. Developing decision support tools for rangeland management by combining state and transition models and Bayesian belief networks.

Agricultural Systems. 99, 23-34.

Cain, J.D., Jinapala, K., Makin, I.W., Somaratna, P.G., Ariyaratna, B.R., Perera, L.R., 2003.

Participatory decision support for agricultural management. A case study from Sri Lanka. Agricultural Systems. 76, 457-482.

Chen, S.H., Pollino, C.A., 2012. Good practice in Bayesian network modelling. Environmental Modelling and Software. 37, 134-145.

Everingham, Y.L., Muchow, R.C., Stone, R.C., Inman-Bamber, N.G., Singels, A., Bezuidenhout, C.N., 2002. Enhanced risk management and decision making capability across the sugar industry value chain based on seasonal climate forecasts. Agricultural Systems 74, 459-477.

Godfray, C.J., Beddington, J.R., Crute, I.R., Haddad, L., Lawrence, D., Muir, J.F., Pretty, J., Robinson, S., Thomas, S.S.M., Toulminlo, C., 2010. Food security: the challenge of feeding 9 billion people. Science 327, 812-817.

Hens, L., Vromant, N., Tho, N., and Hung, N. T., 2009. Salination of surface water, groundwater, and soils in the shrimp farming areas of the coastal Cai Nuoc district, South Vietnam. International Journal of Environmental Studies. 66, 69-81.

Huq, N., Huge, J., Boon, E., Gain, A.K., 2015. Climate Change Impacts in Agricultural Communities in Rural Areas of Coastal Bangladesh: A Tale of Many Stories. Sustainability. 7, 8437-8460. 
Jensen F.V. and Nielsen T.D., 2007. Bayesian Networks and Decision Graphs. Springer, New York, NY.

Joffre, O.M., Bosma, R. H., Ligtenberg, A., Tri, V. P. D., Ha, T. T. P., Bregt, A.K., 2015 Combining participatory approaches and an agent-based model for better planning shrimp aquaculture, Agricultural Systems. 141, 149-159.

Johnson, S., Mengersen, K., de Waal, A., Marnewick, K., Cilliers, D., Houser, A.M., Boast, L., 2010. Modelling cheetah relocation success in southern Africa using an Iterative Bayesian Network Development Cycle. Ecological Modelling. 221, 641-651.

Korb K.B. and Nicholson A.E., 2004. Bayesian Artificial Intelligence. Chapman and Hall, Boca Raton, FL.

Kristjanson, P., Reid, R.S., Dickson, N., Clark, W.C., Romney, D., Puskur, R., MacMillan, S., Grace, D., 2009. Linking international agricultural research knowledge with action for sustainable development. Proceedings of the National Academy of Sciences. 9, 5047-5052.

Kuhnert, P.M., Martin, T.G., Griffiths, S.P., 2010. A guide to eliciting and using expert knowledge in Bayesian ecological models. Ecology Letters. 13, 900-914.

Landuyt, D., Lemmens, P., D'hondt, R., Broekx, S., Liekens, I., De Bie, T., Declerck, S. A. J., De Meester, L., \& Goethals, P. L. M., 2014. An ecosystem service approach to support integrated pond management: A case study using Bayesian belief networks-Highlighting opportunities and risks. Journal of Environmental Management, 145, 79-87.

Leigh, C., Hiep, L.H., Stewart-Koster, B., Vien, D.M., Condon, J., Sang, N.V., Sammut, J., Burford, M.A., 2017. Concurrent rice-shrimp-crab farming systems in the Mekong Delta: Are 
conditions (sub) optimal for crop production and survival? Aquaculture Research, online early, DOI: 10.1111/are.13338

Liedloff, A.C., Woodward, E.L., Harrington, G.A., Jackson, S., 2013. Integrating indigenous ecological and scientific hydro-geological knowledge using a Bayesian Network in the context of water resource development. Journal of Hydrology 499, 177-187.

Norsys (2010) NETICA. Available at: http://www.norsys.com.

Marcot, B.G., Steventon, J.D., Sutherland, G.D., McCann, R.K., 2006. Guidelines for developing and updating Bayesian belief networks applied to ecological modeling and conservation. Canadian Journal of Forest Research. 36, 3063-3074.

McCann, R.K., Marcot, B.G., Ellis, R., 2006. Bayesian belief networks: applications in ecology and natural resource management. Canadian Journal of Forest Research. 36, 30533062.

McDowell, R.W., Nash, D., George, A., Wang, Q.J., Duncan, R., 2009. Approaches for quantifying and managing diffuse phosphorus exports at the farm/small catchment scale. Journal of Environmental Quality. 38, 1968-1980.

Morton, J.F., 2007. The impact of climate change on smallholder and subsistence agriculture. Proceedings of the National Academy of Sciences. 104, 19680-19685.

Phan, T. D., Smart, J. C. R., Capon, S. J., Hadwen, W. L., 2016. Applications of Bayesian belief networks in water resource management: A systematic review. Environmental Modelling \& Software 85, 98-111. 
Pollino, C.A., Woodberry, O., Nicholson, A., Korb, K., Hart, B.T., 2007. Parameterisation and evaluation of a Bayesian network for use in an ecological risk assessment. Environmental Modelling and Software 22, 1140-1152.

Rahman, M.M., Hagare, D., Maheshwari, B., 2015. Framework to assess sources controlling soil salinity resulting from irrigation using recycled water: an application of Bayesian belief network. Journal of Cleaner Production, 105, 406-419.

Reid, P., Vogel, C., 2006. Living and responding to multiple stressors in South Africa glimpses from KwaZulu-Natal. Global Environmental Change. 16, 195-206

Renooij, S., 2001. Probability elicitation for belief networks: issues to consider. The Knowledge Engineering Review. 16, 255-269.

Schmitt, L.H.M., Brugere, C., 2013. Capturing ecosystem services, stakeholders' preferences and trade-offs in coastal aquaculture decisions: a bayesian belief network application. PLoS ONE 8 (10).

Smith, C.S., Russell, I., King, C., 2005. Rats and rice: belief network models of rodent control in the rice field of Cambodia. In: Zerger, A., Argent, R.M. (Eds.), MODSIM 2005 International Congress on Modelling and Simulation. Modelling and Simulation Society of Australia and New Zealand, pp. 449-455.

Stewart-Koster B., Bunn S.E., Mackay S.J., Poff N.L., Naiman R.J., Lake P.S., 2010, The use of Bayesian networks to guide investments in flow and catchment restoration for impaired river ecosystems. Freshwater Biology. 55, 243-260.

Thanh, T., Saito, Y., Huy, D., Nguyen, V., Ta, T., Tateishi, M., 2004, Regimes of human and climate impacts on coastal changes in Vietnam. Regional Environmental Change. 4, 49-62. 
Tho N., Merckx R., Ut V. N., 2012, Biological characteristics of the improved extensive shrimp system in the Mekong delta of Vietnam. Aquaculture Research. 43, 526-537.

Tho N., Ut V. N., Merckx R., 2011, Physico-chemical characteristics of the improved extensive shrimp farming system in the Mekong delta of Vietnam. Aquaculture Research, 42, $1600-1614$.

Tho, N., Vromant, N., Hung, N. T., Hens, L., 2008. Soil salinity and sodicity in a shrimp farming coastal area of the Mekong Delta, Vietnam. Environmental Geology. 54, 1739-1746.

Ticehurst, J.L., Newham, L.T.H., Rissik, D., Letcher, R.A., Jakeman, A.J., 2007. A Bayesian network approach for assessing the sustainability of coastal lakes in New South Wales, Australia. Environmental Modelling and Software. 22, 1129-1139.

Voinov, A., Bousquet, F., 2010. Modelling with stakeholders. Environmental Modelling and Software. 25, 1268-1281.

Walker, D. H., Sinclair, F. L. \& Thapa, B., 1995. Incorporation of indigenous knowledge and perspectives in agroforestry development. Part 1: review of methods and their application. Agroforestry Systems. 30, 235-248. 


\section{Tables}

Table 1. Elicitation approaches used to populate conditional probability tables within the final BBN. Direct methods consist of those where the expert is asked to state their expectation of each state of the child node given certain states of the parent node(s). In direct methods are where the expert is asked a series of related questions to identify where their belief lies about the nodes.

\begin{tabular}{llll}
\hline Method & $\begin{array}{l}\text { Type of } \\
\text { elicitation }\end{array}$ & Number of & Information required \\
& & & \\
\hline Survey method & Direct & $10+$ & Statement of single most \\
Direct elicitation & Direct & $1-3$ & Probable outcome \\
& & & Proportion/frequency/probability \\
Gambling & Indirect & $1-3$ & Odds of changing bet under
\end{tabular}


Table 2. The top five most influential ancestral nodes on the outcome of the two child nodes (risk of rice and shrimp failure), and two additional key nodes in the network (wet season water quality and shrimp survival rate). Entropy reduction is a statistic that summarises the effect of ancestral nodes on the child node of interest, larger values indicate more influence (Marcot et al. 2006).

Nodes Entropy reduction Percent

Child node Risk of rice failure

Wet Season water quality

0.48

36

Soil quality

0.2

14.9

Wet season water salinity

0.12

9.1

Soil salinity

0.10

7.7

Fertiliser regime

0.09

6.8

Child node Wet Season WQ

Soil quality

0.68

48.9

Salinity

0.42

30 
Soil salinity

0.32

22.8

Platform preparation

0.18

12.8

Soil pH

0.17

12

Child node Risk of shrimp failure

Shrimp survival rate

0.56

32.3

Shrimp disease

0.21

12.1

Shrimp stress

0.15

8.7

Lab lab

0.06

3.2

Stress detection timing

0.02

0.9

Child node Shrimp survival rate

Shrimp disease

0.298

28.7

Shrimp stress

0.226

21.7 
Lab lab

Stress detection timing

Shrimp stocking density
0.027

2.58

0.144

13.8

0.027

2.57 


\section{Figures}

a)

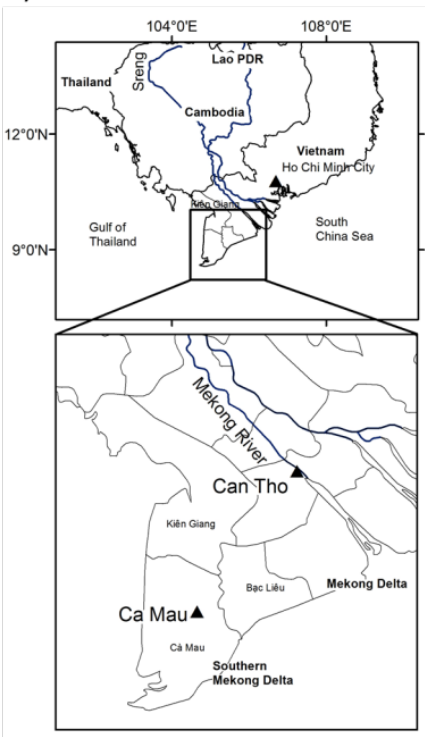

b)

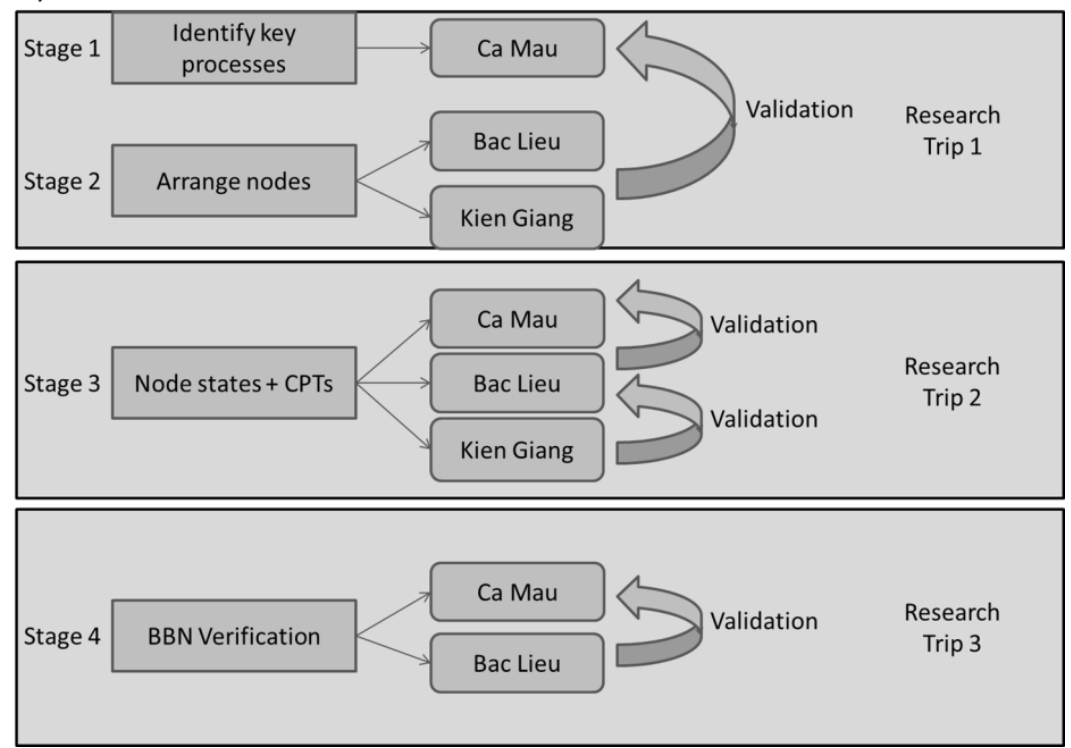

Figure 1. a) Map of the region showing the three provinces on the southern Mekong Delta where the expert elicitation workshops took place. b) Conceptual diagram of staged iterative process expert elicitation process across all three provinces. 

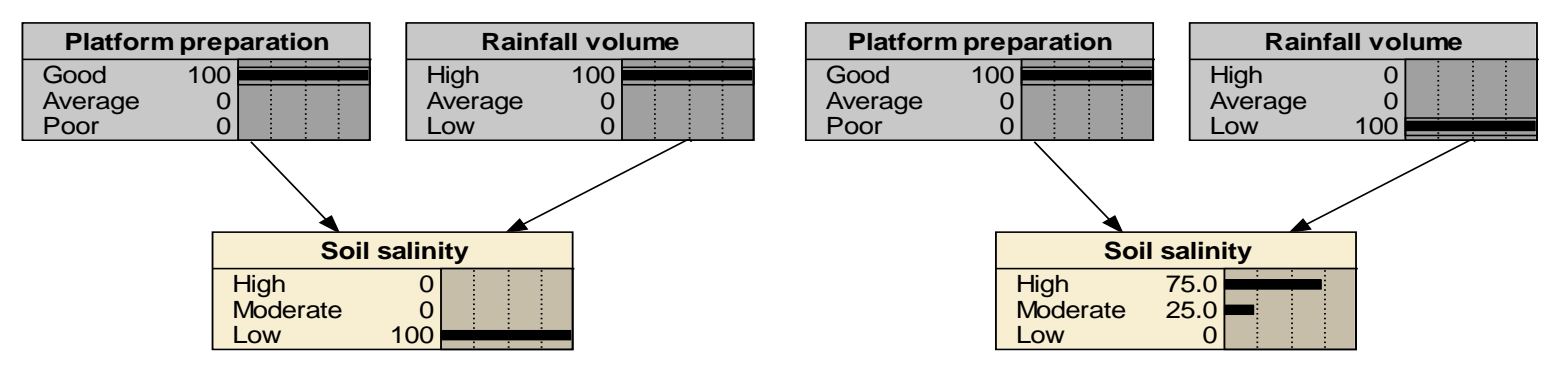

Figure 2. Schematic of BBN showing the change in probability of different states of a child node given different conditions in the parent node. 


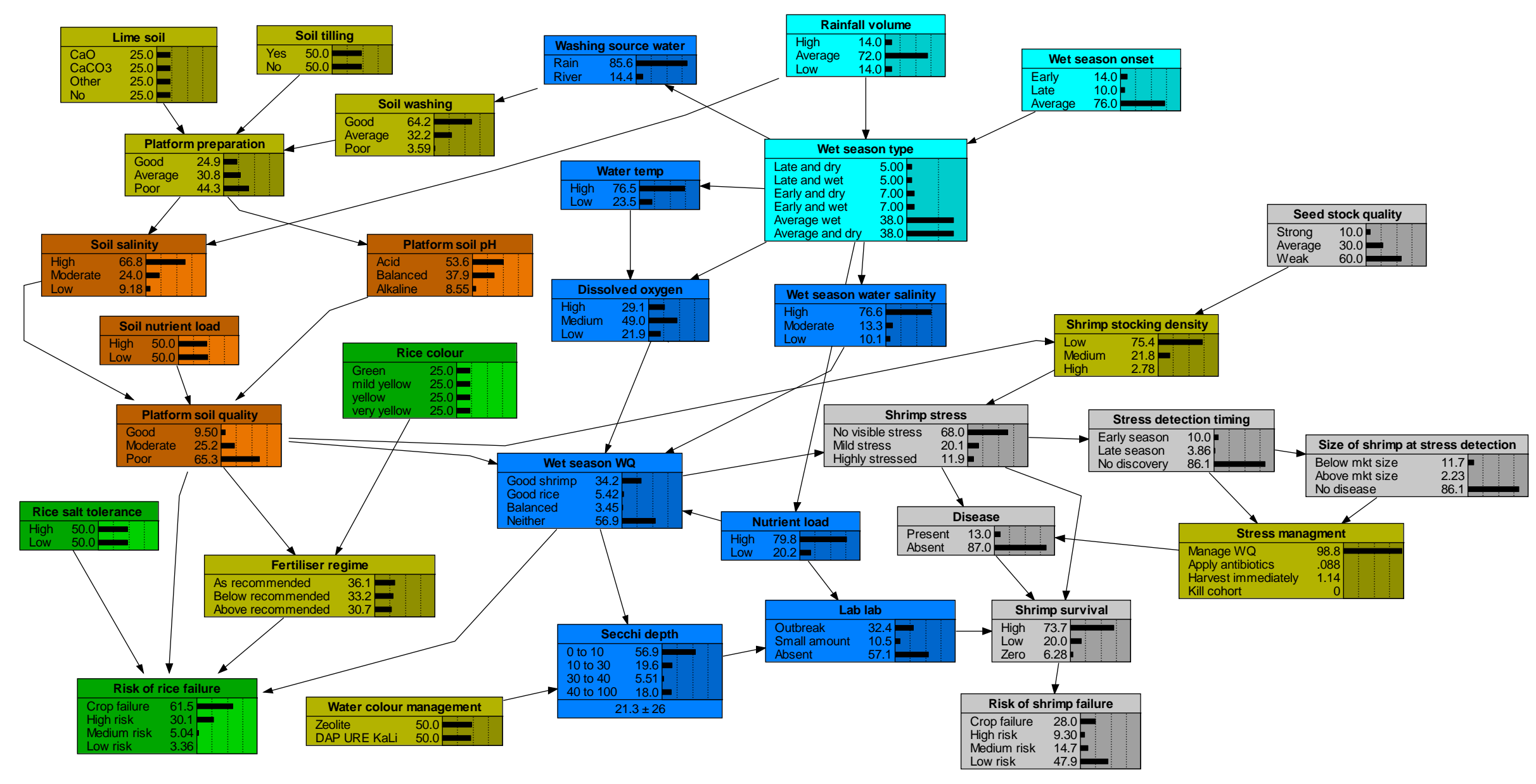

Figure 3. The final BBN representing the knowledge and experience of the participating farmers and extension officers. 
(a)

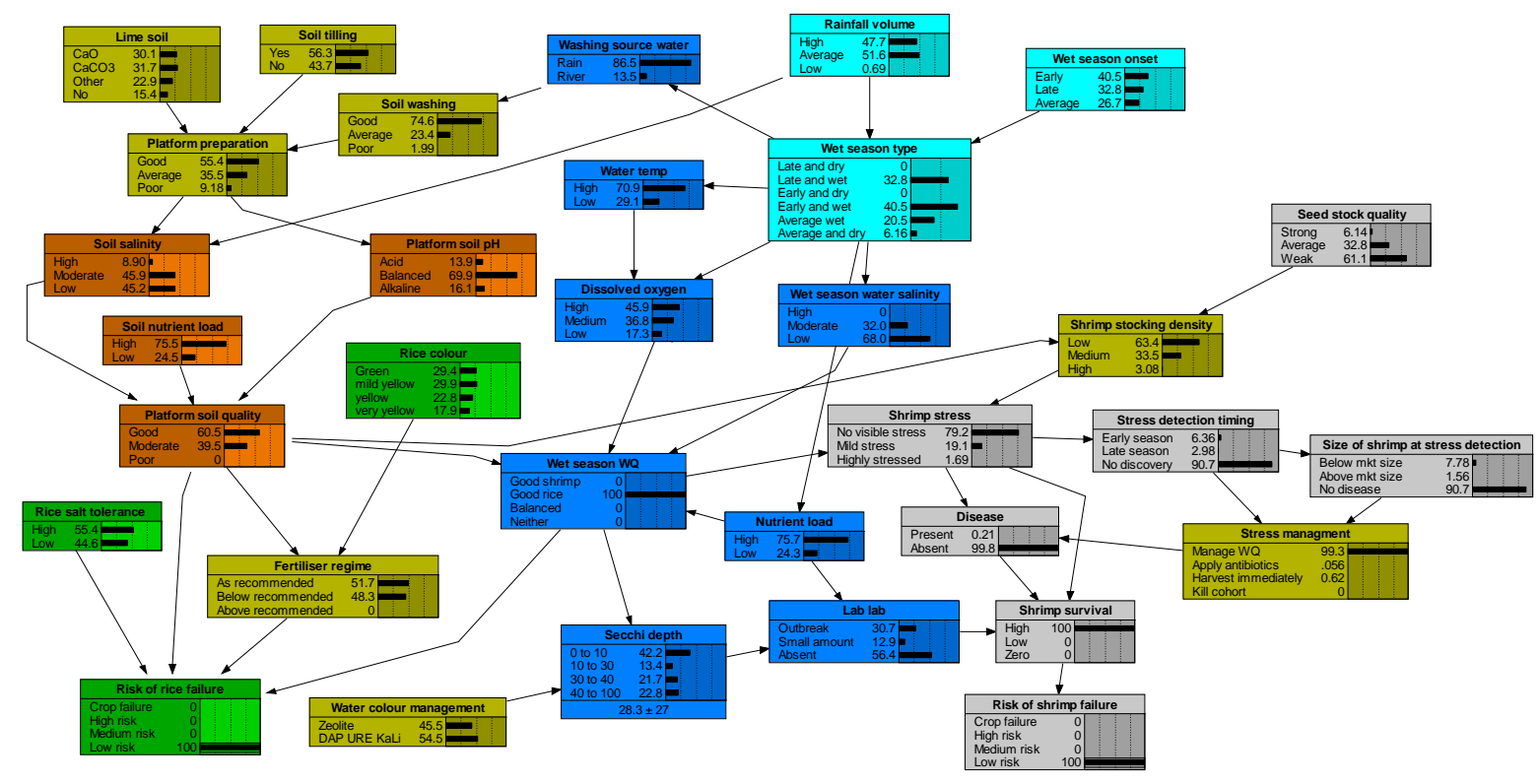

(b)

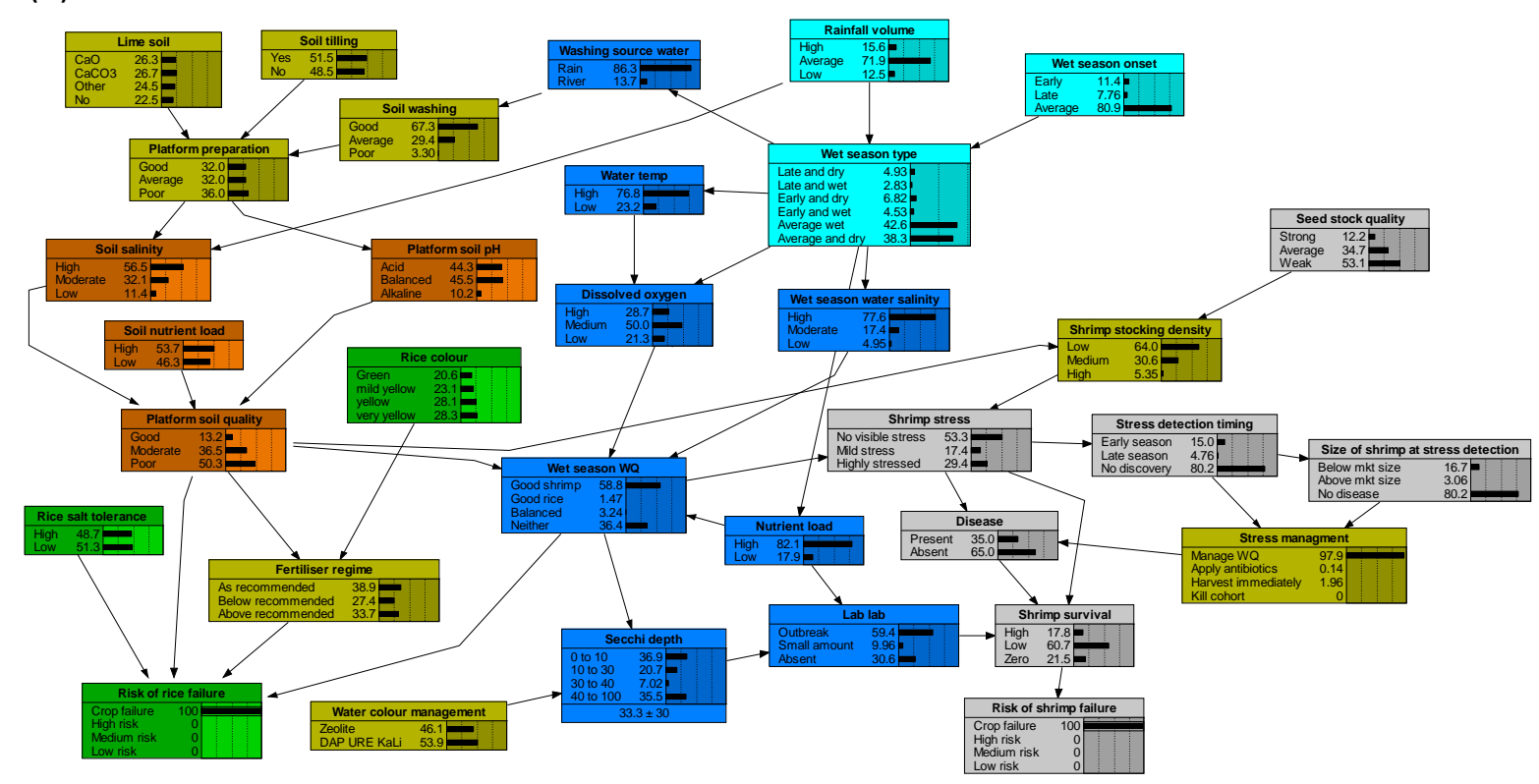

Figure 4. The complete BBN with both child nodes set to (a) low risk of crop failure and (b) complete crop failure. With conditions set in the child nodes, each BBN shows the range of potential scenarios under which these outcomes can occur. 
(a) Water quality $=$ good for rice

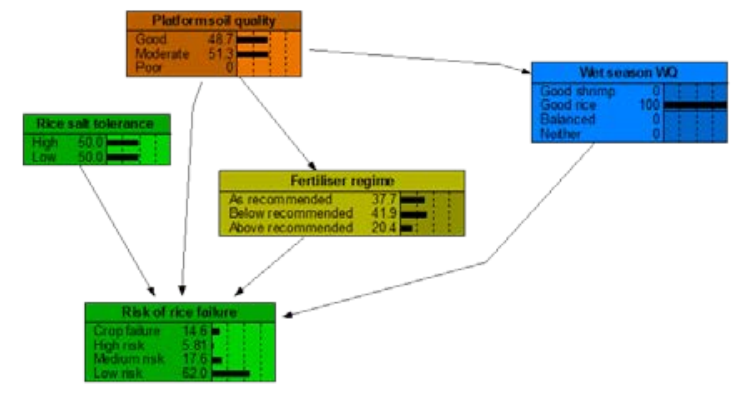

(c) Soil quality $=$ good

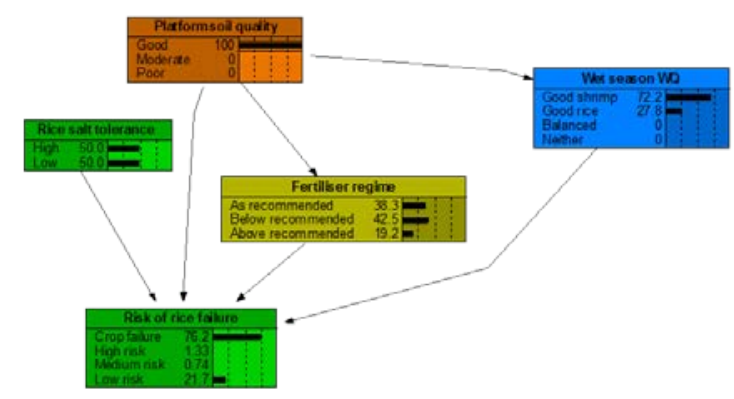

(b) Water quality = good for shrimp

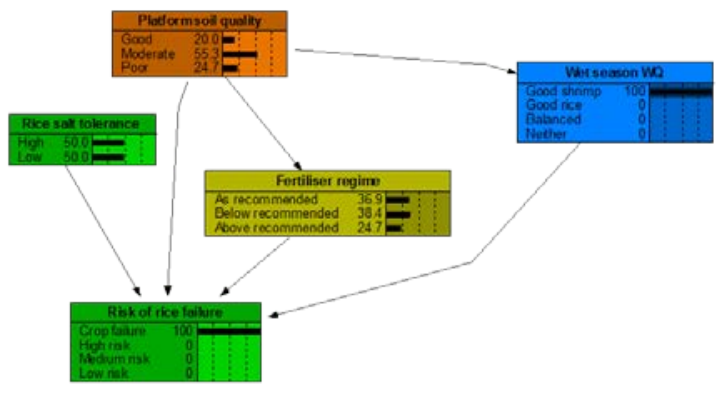

(d) Soil quality $=$ poor

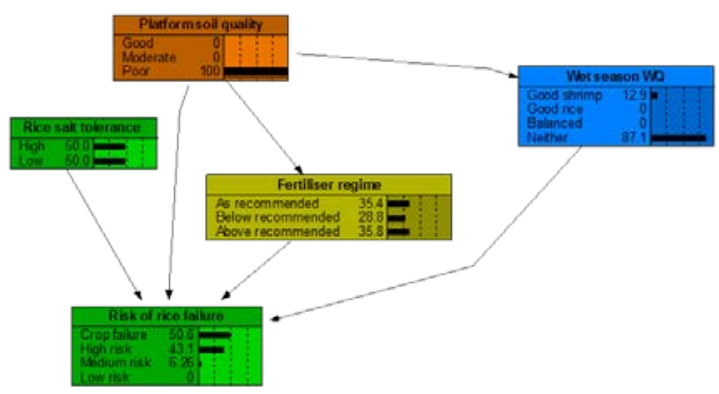

Figure 5. The effect of the most influential parent nodes of the rice node, water quality and soil quality. Changes in these nodes lead to the greatest change in the risk of rice failure. 
(a) Shrimp survival $=$ High

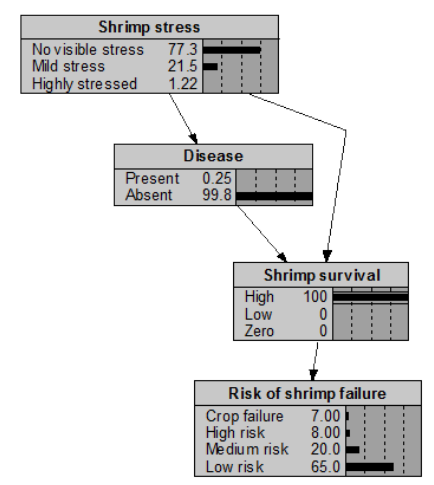

(c) Shrimp disease $=$ present

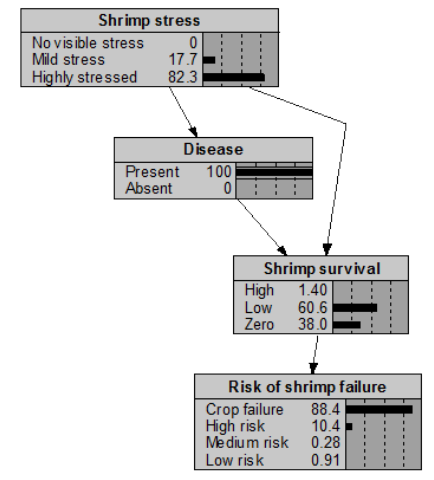

(b) Shrimp survival = Low

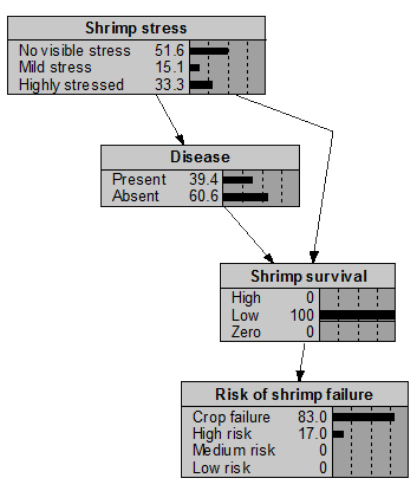

(d) Shrimp disease $=$ absent

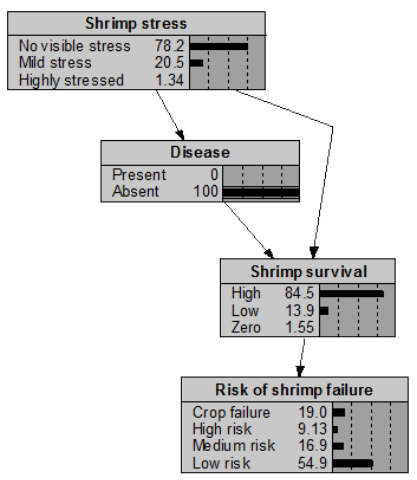

Figure 6. The effect of the most influential parent nodes of the shrimp node, shrimp survival and shrimp disease. Changes in these nodes lead to the greatest change in the risk of shrimp failure 